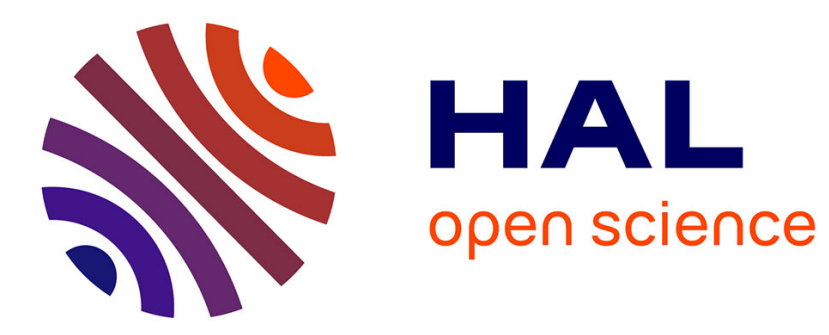

\title{
Precise alignment of a longitudinal Pockels cell for time-resolved circular dichroism experiments
}

\author{
Thibault Dartigalongue, François Hache
}

\section{To cite this version:}

Thibault Dartigalongue, François Hache. Precise alignment of a longitudinal Pockels cell for timeresolved circular dichroism experiments. Journal of the Optical Society of America B, 2003, 20 (8), pp.1780. 10.1364/JOSAB.20.001780 . hal-00836929

\section{HAL Id: hal-00836929}

https://hal-polytechnique.archives-ouvertes.fr/hal-00836929

Submitted on 15 May 2014

HAL is a multi-disciplinary open access archive for the deposit and dissemination of scientific research documents, whether they are published or not. The documents may come from teaching and research institutions in France or abroad, or from public or private research centers.
L'archive ouverte pluridisciplinaire HAL, est destinée au dépôt et à la diffusion de documents scientifiques de niveau recherche, publiés ou non, émanant des établissements d'enseignement et de recherche français ou étrangers, des laboratoires publics ou privés. 


\title{
Precise alignment of a longitudinal Pockels cell for time-resolved circular dichroism experiments
}

\author{
Thibault Dartigalongue and François Hache \\ Laboratoire d'Optique et Biosciences, Centre National de la Recherche Scientifique, Institut National de la Santé et \\ de la Recherche Médicale, Ecole Polytechnique, 91128 Palaiseau cedex, France
}

Received December 6, 2002; revised manuscript received March 19, 2003

\begin{abstract}
Artifacts in time-resolved circular dichroism experiments are carefully analyzed. We show that alignment of the longitudinal Pockels cell that modulates the laser polarization in such experiments is crucial. By developing a calculation of the behavior of a slightly misaligned Pockels cell, we are able to propose a simple alignment procedure. This procedure is based on the use of an analyzer at $0^{\circ}$ or $45^{\circ}$ of the Pockels cell axes, and it allows us to improve the alignment by 3 orders of magnitude and to reduce the artifact below the noise level in our experimental setup. (c) 2003 Optical Society of America
\end{abstract}

OCIS codes: $230.2090,320.7100,320.0320$.

\section{INTRODUCTION}

Time-resolved determination of the conformation of biological molecules has become increasingly important as it appears that many biochemical processes are governed not only by the nature of the molecules in action but also by their spatial configuration. Most of the enzymatic reactions in a living cell can be traced back to conformational changes that significantly alter the chemical properties of molecules. ${ }^{1}$ Such conformational issues are also known to arise in the Alzheimer disease ${ }^{2}$ and in prionrelated pathologies. ${ }^{3}$ Another challenging issue of biochemistry is the folding and unfolding mechanisms in proteins. ${ }^{4}$ Among the techniques that allow one to access such information on a very short (nanosecond or picosecond) time scale, time-resolved circular dichroism (CD) has been proposed several times. We now discuss the principle of the experiment. The CD is the optical property of chiral molecules by which the absorption of light differs for left- or right-circular polarization. As chirality is a fundamental feature of life, CD exists in many biological systems and can therefore serve as a probe of these systems. The principle of a time-resolved CD experiment is to induce a rapid change in the system under study (temperature jump, flash photolysis, laser photoactivation) and to monitor the CD of the perturbed system as a function of time. The problem is to measure the weak CD signal with a high time resolution. Various techniques have been proposed: an ellipsometric method with a strained plate that introduces right and left ellipticity, ${ }^{5,6}$ a splitbeam method, ${ }^{7}$ or laser-induced thermal gratings. ${ }^{8}$ A review of these techniques as well as other related ones can be found in Ref. 9 .

Another more direct approach has been proposed, which is an extension of a commercial CD spectrometer and consists in the development of a standard pumpprobe experiment in which the probe polarization is modulated, alternately circular left and circular right.
The modulation of the probe transmission that is due to $\mathrm{CD}$ is measured by sensitive lock-in detection techniques. $^{10}$ Recently, Mesnil et al. developed a similar approach to study time-resolved CD. ${ }^{11,12}$ Excitation of the molecules was produced with a femtosecond titanium-sapphire laser, and CD measurements were taken by modulation of the probe polarization by application of an alternating voltage on a longitudinal Pockels cell (PC). Use of a PC instead of a photoelastic modulator is dictated by the $1-\mathrm{kHz}$ repetition rate of the laser. This technique has a time resolution of a few hundred femtoseconds and can be straightforwardly extended to biological systems that display CD and can be photoactivated. Demonstration of this technique has been performed with a chiral ruthenium tris(bipyridyl) salt. ${ }^{11,12}$ The experiment was carried out with a linearly polarized pump beam. As extensively investigated in Ref. 10, this feature can induce strong artifacts in the time-resolved CD measurement. A solution is to utilize a depolarized pump beam or to insert a spinning half-wave plate into the pump path. ${ }^{10}$ However, both solutions are sources of complications in the setup and are prone to induce supplementary noise. Here we address the point of using a pump beam with a fixed linear polarization by identifying the conditions for reliable measurements and explaining the experimental procedures that allow us to achieve this goal. As will become clear in Section 2, the crucial point is the alignment of the PC. It is necessary to achieve good circular polarizations and above all to have perfect symmetry between the left and the right circular polarizations of the probe beam. To devise a simple but reliable procedure, we developed a detailed description of the PC and of its interaction with the probe beam. Thanks to this calculation, we have been able to propose a simple alignment procedure that meets our requirements and allows us to obtain CD measurements free from artifacts. 


\section{EXPERIMENTAL ISSUES}

First we give a more detailed description of our experimental setup. We start with an amplified titaniumsapphire laser that functions at $1 \mathrm{kHz}$. This repetition rate is well adapted to pump-probe experiments in liquids: it is slow enough to avoid a heating problem (with the help of a rotating or a circulating cell for our samples, we make sure that the molecules receive only one laser shot) and high enough to permit modulation techniques to be employed. After several stages of optical parametric amplification, we dispose of $5-\mu \mathrm{J}, 180-\mathrm{fs}$ pulses tunable from 470 to $550 \mathrm{~nm}$. These pulses serve as excitation pulses and are well fitted for a whole set of chemical or biological molecules. To obtain a continuously tunable probe, we took a few microjoules of the laser, doubled its frequency to $400 \mathrm{~nm}$, and generated a white-light continuum by focusing the laser in a sapphire plate. The tunability extends from 400 to $500 \mathrm{~nm}$. The pulse energy is weak but easily measurable with a photomultiplier tube (PMT).

To measure the CD, we introduced a longitudinal PC in the probe path. The purpose of this PC is to modulate the light polarization alternately circular left and circular right. To achieve this, we applied a square voltage on the PC: $\pm(1300-1500) \mathrm{V}$ at $80 \mathrm{~Hz}$. The voltage was chosen so that the PC functioned as a quarter-wave plate for the probe. The precise value is a function of the probe wavelength. The choice of the modulation frequency involves several constraints: it must be low enough compared with the $1-\mathrm{kHz}$ repetition rate of the laser and as high as possible to reduce the $1 / f$ noise. At the same time, it must be well separated from the noise sources of our experiments (vibrations, line voltage...). The principle of the experiment is as follows. Because of the CD, the transmission of the sample depends on the handedness of the light, and the signal measured by the PMT is modulated at the PC frequency. This modulation is extracted from the signal with the help of a lock-in amplifier.

Let us call $\alpha=\alpha_{0} \pm \delta \alpha$ the absorption of the left and right circularly polarized light. The transmission of the sample, as measured with the PMT, reads

$$
T=T_{0} \exp (-\alpha L),
$$

where $T_{0}$ stands for the sample independent parameters (reflection losses, filters, PMT efficiency) and $L$ is the cell thickness. In a general case when $\delta \alpha \ll \alpha_{0}$, Eq. (1) becomes

$$
T=T_{0} \exp \left(-\alpha_{0} L\right)(1 \mp \delta \alpha L) .
$$

The modulated part of $T$ is $T_{0} \exp \left(-\alpha_{0} L\right) \delta \alpha L$, and one can determine that the $\mathrm{CD} \equiv 2 \delta \alpha L$ is straightforwardly given by the ratio of the lock-in signal over the PMT signal.

The complete experimental setup is sketched in Fig. 1. A linearly polarized pump pulse impinges on the sample and triggers the process that we want to study. The probe beam is then sent onto the sample with a variable delay $\tau$. Both beams are focused on the sample ( $f$ $=250 \mathrm{~mm}$ ). The transmitted signal is measured together with the PMT and the lock-in amplifier, allowing determination of the CD. The experiment consists in

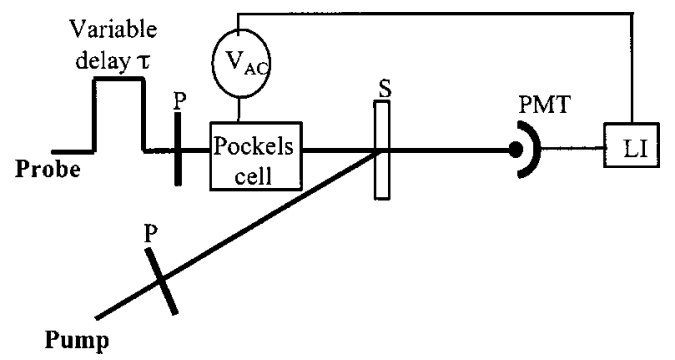

Fig. 1. Experimental setup for time-resolved circular dichroism measurements. The pump is linearly polarized, whereas the probe polarization is modulated alternatively circular left or right by the Pockels cell: P, polarizers; S, sample; LI, lock-in amplifier.

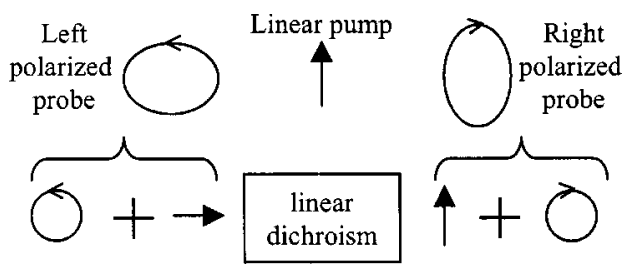

Fig. 2. Qualitative illustration of the linear dichroism that can occur if the probe polarizations are not perfectly left or right circular.

measuring the CD as a function of delay $\tau$. Given that we have 150 -fs pulses, we were able to monitor changes in the CD on this time scale and therefore to follow conformational changes with unprecedented accuracy.

As depicted above, the experimental principle is simple. However, this is not the whole story, as one must make sure that the measured signal is the expected one and that it is free of artifacts. In such an experimental configuration in which two beams interact, one with linear polarization and the other with circular polarization, this is challenging because artifacts are common. A qualitative discussion of these artifacts has been thoroughly reported in Refs. 9 and 10. Suppose that the left and right circular polarizations of the probe are not perfect and that they are in reality slightly elliptically polarized, but with different axes of ellipticity, as depicted in Fig. 2. These polarizations can be decomposed into a perfect circular polarization plus a small linear polarization. Now, depending on whether the direction of the small linear polarization is parallel or perpendicular to the pump polarization, it will lead to different effects, known as pumpinduced linear dichroism. In our setup this translates into a modulation of the probe transmission, which has nothing to do with a pump-induced CD signal. Given that the CD signals are usually weak, these artifacts can rapidly overcome the expected signals.

We now describe these artifacts in a more precise manner so as to be able to define criteria that allow us to trust our experimental results. For that purpose, let us consider the propagation of an electromagnetic wave in an achiral medium. We write the probe electric field $E(Z, t)=A(Z) \exp (-i \omega t)+$ c.c., where $Z$ is the propagation direction. The equation that describes the evolution of $A$ in the presence of a pump (intensity $I_{p}$ ) is

$$
\frac{\partial A}{\partial Z}=-\frac{1}{2}\left(\alpha+\beta I_{p}\right) A .
$$


In Eq. (3) we introduced the pump-induced absorption $\beta I_{p}$. The important point for our discussion is that $\beta$ assumes different values, depending on whether the pump and probe polarizations are parallel or perpendicular. We therefore introduce two coefficients $\beta_{\|}$and $\beta_{\perp}$ for these two cases. Denoting $X$ as the direction of the pump polarization, these coefficients can be expressed as a function of the imaginary part of the Kerr nonlinear susceptibility $\chi^{(3)}\left(\omega_{p},-\omega_{p}, \omega\right)$ :

$$
\begin{aligned}
& \beta_{\|}=\frac{\omega}{\left(\epsilon_{0} c n\right)^{2}} \operatorname{Im} \chi_{X X X X}^{(3)}, \\
& \beta_{\perp}=\frac{\omega}{\left(\epsilon_{0} c n\right)^{2}} \operatorname{Im} \chi_{X Y Y X}^{(3)},
\end{aligned}
$$

where $\epsilon_{0}$ is the vacuum permittivity, $c$ is the light speed, and $n$ is the refractive idex. In this formulation we neglected the light-induced change of the refractive index (proportional to the real part of the Kerr susceptibility), which is justified in our resonant experiment in which the pump is absorbed by the sample. The description in terms of Kerr susceptibility might be less adequate than in terms of response functions to describe a time-resolved pump-probe experiment. The following calculation can be easily extended to response functions, but, for the sake of readability, we prefer to stick to the Kerr susceptibility formalism that leads to the same conclusions as far as polarizations are concerned.

Resolution of Eq. (3) for the intensity $\left(\propto|A|^{2}\right)$ of the electric field at the output of the sample yields

$$
I(L)=I(0) \exp (-\alpha L) \exp \left(-\beta I_{p} L_{\text {eff }}\right),
$$

where $\beta$ represents the appropriate $(\|$ or $\perp)$ coefficient and $L_{\text {eff }}=[1-\exp (-\alpha L)] / \alpha$ accounts for the pump beam absorption loss. To describe our experimental problem, we now suppose that the probe is alternately left or right circularly polarized and that the signal we measure is the difference of the corresponding transmissions. To evaluate this, we decompose the circular polarizations in terms of linear polarizations:

$$
\hat{e}_{L, R}=\frac{\exp ( \pm i \varphi)}{\sqrt{2}}(\hat{X} \pm i \hat{Y}) .
$$

The $\exp ( \pm i \varphi)$ terms were included for the sake of completeness: they allow the coincidence of two circular polarizations to take place along any direction and not uniquely along the $X$ direction. It is straightforward to check that the transmission of the sample is the same for the two circular polarizations,

$$
T_{L, R}=\frac{\exp (-\alpha L)}{2}\left[\exp \left(-\beta_{\|} I_{p} L_{\mathrm{eff}}\right)+\exp \left(-\beta_{\perp} I_{p} L_{\mathrm{eff}}\right)\right],
$$

and that, as expected for this achiral medium, there is no CD signal. We now examine what happens if the two circular polarizations are not perfect. We therefore write the two probe electric fields as

$$
A_{L}=A(0)\left(\hat{e}_{L}+\epsilon_{L} \hat{e}_{R}\right),
$$

$$
A_{R}=A(0)\left(\epsilon_{R} \hat{e}_{L}+\hat{e}_{R}\right),
$$

where $\epsilon_{L, R}$ are complex numbers. If the polarizations are close to circular ones, the moduli of $\epsilon_{L, R}$ are small and we retain only first-order terms in our calculation. We can calculate the difference in transmission for the two polarizations:

$$
\begin{aligned}
T_{L}-T_{R}= & {\left[\left(\epsilon_{L}^{\prime}-\epsilon_{R}^{\prime}\right) \cos 2 \varphi+\left(\epsilon_{L}^{\prime \prime}+\epsilon_{R}^{\prime \prime}\right)\right.} \\
& \times \sin 2 \varphi] \exp (-\alpha L)\left[\exp \left(-\beta_{\|} I_{p} L_{\text {eff }}\right)\right. \\
& \left.-\exp \left(-\beta_{\perp} I_{p} L_{\text {eff }}\right)\right] .
\end{aligned}
$$

Several points are worth mentioning. First one sees that this signal is proportional to

$\exp \left(-\beta_{\|} I_{p} L_{\text {eff }}\right)-\exp \left(-\beta_{\perp} I_{p} L_{\text {eff }}\right)$

$$
\propto \operatorname{Im} \chi_{X X X X}^{(3)}-\operatorname{Im} \chi_{X Y Y X}^{(3)} .
$$

This is the expected signature of the pump-induced linear dichroism. ${ }^{13}$ Second we determined that there is no signal if the polarizations are perfectly circular $\left(\epsilon_{L}=\epsilon_{R}\right.$ $=0$ ). On the other hand, Eq. (11) allows us to show that it is possible to cancel the signal if the condition

$$
\epsilon_{L}=\epsilon_{R}^{*}
$$

is satisfied. This implies that, even with slightly elliptical polarizations, one can cancel signal artifacts on the condition that the two ellipses be symmetrical by time reversal. For our present purpose, Eq. (12) provides us with a quantitative criterion for alignment of the PC.

\section{EFFECTS OF A MISALIGNMENT OF THE POCKELS CELL}

We describe the precise behavior of our longitudinal PC when the alignment is not perfect: we suppose that the propagation direction of the laser beam is not perfectly along the PC axis. In Subsection 3.A we calculate the principal polarization directions and their corresponding refractive indices in a general case and then apply this calculation to our experimental configuration in which an alternative voltage is applied to the PC. We are then able to gain a precise description of the two polarization states that emerge from the PC and to apprehend the constraints on the alignment of the PC brought about by Eq. (12).

\section{A. Description of the Pockels Cell}

Let us consider a PC based on a $\mathrm{KD}^{*} \mathrm{P}$ crystal. In the absence of applied voltage, it is a uniaxial crystal with $z$ as its axis. In the case of a longitudinal PC, the voltage is applied along the $z$ axis and, because of the electro-optic effect, the PC becomes biaxial. Denoting $x, y$ as its principal axes, the refractive indices are

$$
\begin{aligned}
& n_{x}=n_{o}+\frac{1}{2} n_{o}^{3} r_{63} V_{z}=n_{o}+\Delta n^{\mathrm{eo}}, \\
& n_{y}=n_{o}-\frac{1}{2} n_{o}^{3} r_{63} V_{z}=n_{o}-\Delta n^{\mathrm{eo}}, \\
& n_{z}=n_{o}+\Delta n^{n},
\end{aligned}
$$


where $\Delta n^{n}=n_{e}-n_{0}$ describes the natural birefringence of $\mathrm{KD}^{*} \mathrm{P}$ and $\Delta n^{\text {eo }}=\frac{1}{2} n_{o}^{3} r_{63} V_{z}$ is the electro-optic birefringence that depends on applied voltage $V_{z}$ and on the electro-optic coefficient. At this point it is good to compare the order of magnitude of the two birefringences. The natural birefringence of $\mathrm{KD}^{*} \mathrm{P}$ is approximately 0.04 in the visible. ${ }^{14}$ On the other hand, we used the PC as a quarter-wave plate in the visible, which means that $(L$ $=2 \mathrm{~cm})$

$$
\Delta \Phi \equiv 2 \Delta n^{\mathrm{eo}} \frac{\omega}{c} L \simeq \frac{\pi}{2} \Rightarrow \Delta n^{\mathrm{eo}} \sim 10^{-5} .
$$

We therefore determined that $\Delta n^{\text {eo }} \ll \Delta n^{n}$ : the induced birefringence is always much smaller than the natural one.

We now consider the laser beam that impinges on the PC. If everything were perfect, the light wave vector $\mathbf{k}$ would be along the PC $z$ axis. However, here we are interested in the effect of a slight misalignment and we suppose that $k_{x}, k_{y} \ll 1$ and $k_{z} \approx 1$ (here we consider unit vector $\hat{k}$ ). Introducing polar angles $\theta$ and $\phi$ (see Fig. 3), we have

$$
\begin{aligned}
& k_{x}=\sin \theta \cos \phi \sim \theta \cos \phi, \\
& k_{y}=\sin \theta \sin \phi \sim \theta \sin \phi, \\
& k_{z}=\cos \theta \sim 1 .
\end{aligned}
$$

In accordance with our hypothesis, $\theta \ll 1$, whereas $\phi$ can assume any value between 0 and $2 \pi$.

The problem now is to determine the principal polarizations for this wave-vector direction. This is a classical problem of crystal optics and the procedure is well known. ${ }^{15}$ One must calculate the ellipse obtained as the cross section of the index ellipsoid by the plane orthogonal to $\mathbf{k}$. The principal directions are given by the short and long axes of the ellipse and the refractive indices by the length of these axes. We start with $(\theta, \phi)$, the parameters that characterize the wave-vector direction. Let us write $M\left(n, \theta^{\prime}, \phi^{\prime}\right)$, the points of the ellipse that we wish

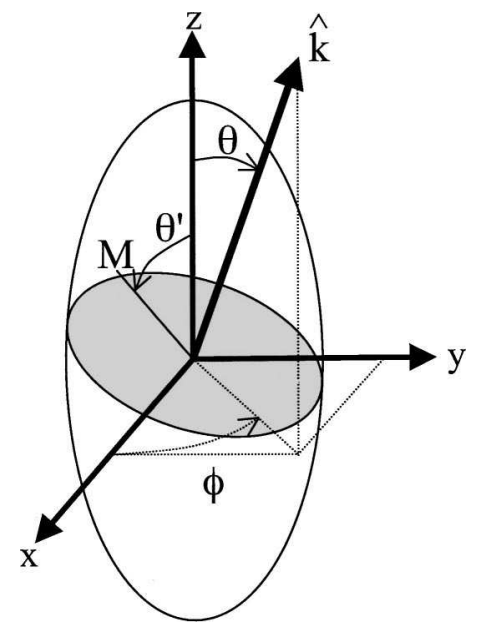

Fig. 3. Representation of the index ellipsoid of the PC. The wave-vector direction $\hat{k}$ is characterized by $\theta$ and $\phi$. The gray ellipse is the cross section of the index ellipsoid by the plane orthogonal to $\hat{k}$; its points $M$ are described by $\theta^{\prime}$ and $\phi^{\prime}$ (not shown). to examine (see Fig. 3). On the one hand, considering that $\theta^{\prime} \sim(\pi / 2)$, the orthogonality condition with $\mathbf{k}$ yields

$$
\theta^{\prime}-(\pi / 2)=\theta \cos \left(\phi-\phi^{\prime}\right) .
$$

On the other hand, points $M$ belong to the index ellipsoid

$$
\frac{\sin ^{2} \theta^{\prime} \cos ^{2} \phi^{\prime}}{n_{x}^{2}}+\frac{\sin ^{2} \theta^{\prime} \sin ^{2} \phi^{\prime}}{n_{y}^{2}}+\frac{\cos ^{2} \theta^{\prime}}{n_{z}^{2}}=\frac{1}{n^{2}} .
$$

Up to first-order birefringences, this equation reads

$$
n=n_{o}+\Delta n^{\mathrm{e} o} \sin ^{2} \theta^{\prime} \cos 2 \phi^{\prime}+\Delta n^{n} \cos ^{2} \theta^{\prime},
$$

which, when plugging in to Eq. (16), yields to the lowest order in $\theta$,

$$
n=n_{o}+\Delta n^{\text {eo }} \cos 2 \phi^{\prime}+\Delta n^{n} \theta^{2} \cos ^{2}\left(\phi-\phi^{\prime}\right) .
$$

In Eq. (19) we took into acount the fact that $\Delta n^{\text {eo }}$ $\ll \Delta n^{n}$. This equation expresses the value of the refractive index for a polarization characterized by an azimuthal angle $\phi^{\prime}$ [ $\theta^{\prime}$ being determined by Eq. (16)]. The next step to obtain the principal polarizations and indices is to calculate the extremum values of this index and to determine the corresponding angle. Before going to the general case, we can check the pertinence of this equation in the limiting cases. If there is no voltage applied to the PC $\left(\Delta n^{\mathrm{eo}}=0\right)$, we obtain the two principal directions of $\phi^{\prime}=\phi$ and $\phi^{\prime}=\phi+\pi / 2$ as expected for a uniaxial crystal, and the corresponding birefringence is

$$
\Delta n_{V=0}=\Delta n^{n} \theta^{2},
$$

independent of $\phi$. This particular point will be discussed later to a greater extent as it corresponds to the conventional alignment procedure of a PC between two crossed polarizers. As another limiting case, we consider a PC with an applied voltage for perfect alignment $(\theta=0)$. Equation (19) reads as $n=n_{o}+\Delta n^{\mathrm{eo}} \cos 2 \phi^{\prime}$, and the principal directions are $\phi^{\prime}=0$ and $\phi^{\prime}=\pi / 2$, corresponding to the $x$ and $y$ axes of the PC, as expected.

We now come to the general solution. Zeroing the derivative of Eq. (19) with respect to $\phi^{\prime}$ yields an implicit equation:

$$
\sin \left(2 \phi^{\prime}\right)=\frac{\Delta n^{n}}{2 \Delta n^{\mathrm{eo}}} \theta^{2} \sin 2\left(\phi-\phi^{\prime}\right) .
$$

As we are interested in a slight misalignment, we expect $\phi^{\prime}$ to be close to 0 (modulo $\pi / 2$ ), and we determined that the two solutions of Eq. (21) are $\phi^{\prime}=\alpha$ and $\phi^{\prime}=\alpha$ $+\pi / 2$ with

$$
\alpha=\frac{\Delta n^{n}}{4 \Delta n^{\mathrm{eo}}} \theta^{2} \sin 2 \phi .
$$

This solution is correct as long as $\theta^{2}<\left(2 \Delta n^{\mathrm{eo}}\right) /\left(\Delta n^{n}\right)$, which sets a limit of a few $10^{-4}$ on $\theta^{2}$. We can rewrite Eq. (22) by reintroducing the wave-vector direction

$$
\alpha=\frac{\Delta n^{n}}{2 \Delta n^{\mathrm{eo}}} k_{x} k_{y} .
$$

Equation (23) together with Eq. (16) fully determines the direction of the principal polarizations. We obtained the 
corresponding refractive indices by plugging $\alpha$ into Eq. (19). We obtained the actual birefringence $\Delta n^{\text {eff }} \equiv n(\alpha)$ - $n(\alpha+\pi / 2)$ undergone by the laser beam, which reads, to the lowest order in $\theta$,

$$
\Delta n^{\mathrm{eff}}=2 \Delta n^{\mathrm{eo}}+\Delta n^{n} \theta^{2} \cos 2 \phi,
$$

which we can rewrite in terms of the wave-vector direction

$$
\Delta n^{\text {eff }}=2 \Delta n^{\text {eo }}+\Delta n^{n}\left(k_{x}^{2}-k_{y}^{2}\right) .
$$

\section{B. Application of the Alternative Voltage: States of Polarization of the Outcoming Laser in a General Case}

To continue with our experiments, we now apply an alternative symmetrical voltage $\pm \mathrm{V}$ to the $\mathrm{PC}$. First, we must analyze the changes brought about by the change in sign. In the above equations, this amounts to a change in sign of the electro-optic birefringence that is now $\pm \Delta n^{\mathrm{eo}}$, with the other (geometric) parameters remaining unchanged. The subsequent changes are twofold. On the one hand, angle $\alpha$ changes sign [see Eq. (23)]. This means that the direction of the principal polarizations changes when the voltage sign is changed. On the other hand, the effective birefringences are no longer symmetrical: only the first term on the left-hand side of Eq. (25) changes sign whereas the second term does not. This rapid examination of the above equations allows us to understand that a misalignment of the PC rapidly induces dissymmetry between the two polarizations that will perturb the experiments.

With the information in Subsection 3.A, we can be even more precise and treat the general case for which, in addition to the PC misalignment, other experimental parameters are not perfectly matched. First, we note that, under normal use, the polarization of the beam that impinges on the $\mathrm{PC}$ is linear and at $\pi / 4$ of the $x$ and $y$ axes of the PC. Here we suppose that this adjustment is not perfect and that the angle between the laser polarizarion and the $x$ axis is $\pi / 4+s$. As seen above, misalignment of the PC implies that the angle between the input polarization and the principal polarization directions of the PC are actually $\pi / 4+s \mp \alpha$ and $3 \pi / 4+s \mp \alpha$ depending on the voltage sign. Second, the positive and negative voltages applied to the PC might not be perfectly matched to the quarter-wave voltage of the PC. To account for this, we write the total electro-optic dephasings as $\varphi^{ \pm}$

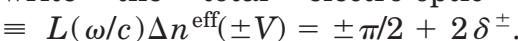

We now examine the polarization of the outcoming beam for the positive voltage. Writing $\hat{x}_{\alpha}$ and $\hat{y}_{\alpha}$, the principal polarization directions, the input beam reads

$$
E_{\text {in }}=\cos \left(\frac{\pi}{4}+s-\alpha\right) \hat{x}_{\alpha}+\sin \left(\frac{\pi}{4}+s-\alpha\right) \hat{y}_{\alpha} .
$$

After propagation in the $\mathrm{PC}$, the two components are dephased by $\varphi^{+}$. By keeping only first-order terms in $s$, $\alpha$ and $\delta^{+}$, we obtain

$$
\begin{aligned}
& E_{\text {out }}^{+}= \frac{\exp \left(i \delta^{+}\right)}{\sqrt{2}}\left[(1-s+\alpha)\left(1-i \delta^{+}\right) \hat{x}_{\alpha}\right. \\
&\left.+i(1+s-\alpha)\left(1+i \delta^{+}\right) \hat{y}_{\alpha}\right] .
\end{aligned}
$$

Introducing the circular polarizations $\hat{e}_{L, R}=(1 / \sqrt{2})$ $\times\left(\hat{x}_{\alpha} \pm i \hat{y}_{\alpha}\right)$, we can rewrite Eq. (27) (to within a global phase factor)

$$
E_{\text {out }}^{+}=\hat{e}_{L}+\left(\alpha-s-i \delta^{+}\right) \hat{e}_{R} .
$$

A similar calculation for the negative voltage yields

$$
E_{\text {out }}^{-}=\hat{e}_{R}+\left(-\alpha-s-i \delta^{-}\right) \hat{e}_{L} .
$$

We therefore obtained the expected results: for the positive and negative voltages, the output polarization is, respectively, left and right circular with a small right and left circular component that arises from the various sources of misalignment. One should be careful here because the two sets of circular polarizations are not strictly identical and depend on the voltage sign. Taking this difference into account would bring higher-order terms and can therefore be neglected. We can now utilize directly the results of Section 2 with

$$
\begin{aligned}
\epsilon_{L} & =\alpha-s-i \delta^{+}, \\
\epsilon_{R} & =-\alpha-s-i \delta^{-} .
\end{aligned}
$$

The condition $\epsilon_{L}=\epsilon_{R}^{*}$ yields

$$
\begin{aligned}
\alpha & =0, \\
\delta^{+}+\delta^{-} & =0 .
\end{aligned}
$$

With the help of Eqs. (23) and (25) we can reexpress these conditions as functions of the wave-vector direction:

$$
\begin{gathered}
\alpha=\frac{\Delta n^{n}}{2 \Delta n^{\mathrm{eo}}} k_{x} k_{y}=0, \\
\delta^{+}+\delta^{-}=\frac{L \omega}{c} \Delta n^{n}\left(k_{x}^{2}-k_{y}^{2}\right)=0 .
\end{gathered}
$$

Several points are worth mentioning. First, we observed that angle $s$ does not come into play in these equations (as long as it is small enough). This is a convenient feature: the PC does not need to be exactly at $45^{\circ}$ in our experiment. If the angle differs slightly from $45^{\circ}$, it induces a small ellipticity in the polarizations, but the two polarizations are still symmetrical and this does not induce an artifact signal. Similarily, $\Delta n^{\mathrm{e} o}$ does not appear in Eq. (35), which means that the exact values of the applied voltages are not so important. If the latter differ from the quarter-wave voltages, it also induces some ellipticity in the polarization but no artifacts. Examining Eqs. (34) and (35), one sees that the only important feature to eliminate artifacts is the alignment of the PC. Indeed, one must have $k_{x}$ and $k_{y}$ identically zero to satisfy both equations. It therefore appears that alignment of the PC is even more crucial than expected: there is no way to obtain symmetrical circular polarizations if the PC is not perfectly aligned. Fortunately, the above calculations provide us with a thorough understanding of the role of the various parameters and allow us to design an alignment procedure that we detail in Section 4. 


\section{ALIGNMENT PROCEDURE OF THE POCKELS CELL}

\section{A. Preliminary Alignment}

To obtain the results presented in Section 3, we supposed that the misalignment was weak enough to keep only the lowest-order terms. The first requirement is therefore to perform a preliminary adjustment that allows this hypothesis to be valid. This is quite readily done with the conventional alignment procedure of a PC. The setup consists of a polarizer at $45^{\circ}$ of the PC axes, a diffusing plate, the PC with no applied voltage, and an analyzer crossed with the polarizer. The role of the diffusing plate is to give rise to a large range of wave-vector directions. We can apply the results of Section 3. For a given direction of the wave vector, characterized by angle $\phi$ (whatever the angle $\theta$ ), the principal polarization directions are $\phi$ and $\phi+\pi / 2$. As a consequence, the beams whose misalignment is in the direction of the polarizer axis or in the direction of the analyzer axis remain unchanged and are blocked by the analyzer, which gives rise to a dark cross (see Fig. 4). Now when we take into consideration the other directions of propagation, the beams undergo a dephasing given by $\Delta n_{V=0}(2 \pi L / \lambda)$ [see Eq. (20)], which causes the polarization to become elliptic. When this dephasing is equal to a multiple of $2 \pi$, we again observed an extinction of the light. The condition for the first extinction can be written as

$$
k_{x}^{2}+k_{y}^{2}=\frac{\lambda}{L \Delta n^{n}},
$$

and one can observe a series of concentric dark circles, as are visible in Fig. 4. By placing the direct laser beam (without the diffusing plate) at the center of this figure, one can easily achieve good precision for the wave-vector direction. The validity condition of the above calculations $\left[\theta^{2} \sim 10^{-4}\right.$; see Eq. (22)] is satisfied as soon as the laser spot is at a distance from the center that is smaller than half of the radius of the first dark circle. We can also estimate the precision that one can achieve with this classical procedure. If we align the laser spot with a pre-

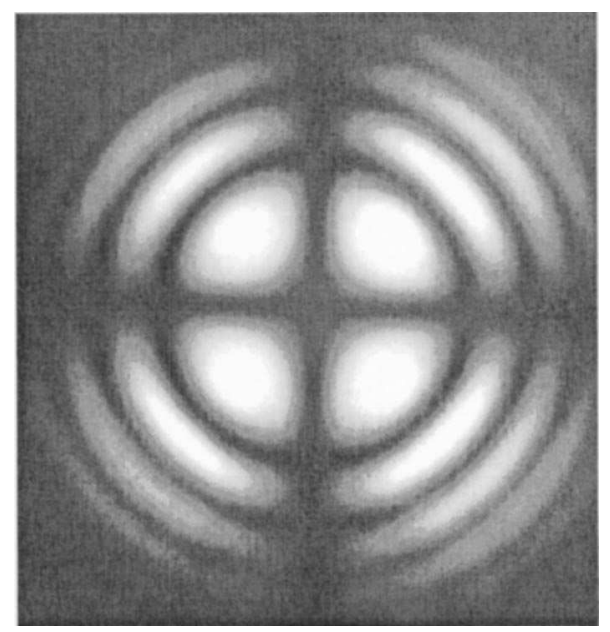

Fig. 4. Photography of the structure observed in the preliminary alignment of the PC (see text). The dark cross and the concentric circles are clearly observable. cision of $1 / 10$ of the dark circle radius, we would obtain $\theta^{2} \sim 5 \times 10^{-6}$. It is possible to enhance this precision as demonstrated below.

\section{B. Adjustment Procedure}

To obtain alignment of the PC that is compatible with our experiments, we must go beyond this simple alignment. We use a setup with a polarizer, a $\mathrm{PC}$ with the alternative voltage on, and an analyzer, but we now allow the angle of the analyzer to vary. We refer to the analyzer angle as $a$ compared with the PC $x$ axis. The light beams that impinge onto the analyzer are given by Eqs. (28) and (29). After the beams pass through the analyzer, the light intensities can be calculated to be

$$
I^{ \pm} \propto 1+2( \pm \alpha-s) \cos 2 a \mp \delta^{ \pm} \sin 2 a .
$$

This means that the signal is modulated at the modulation frequency of the applied voltage. Measuring this modulation with a lock-in (LI) amplifier yields signal

$$
\mathrm{LI} \propto 4 \alpha \cos 2 a-\left(\delta^{+}+\delta^{-}\right) \sin 2 a .
$$

This formula allows us to control the alignment of the PC: if we adjust the analyzer angle to $0^{\circ}$ or $45^{\circ}$, the LI signal depends only on $\alpha$ or $\delta^{+}+\delta^{-}$. Figure 5 represents the $\left(k_{X}, k_{Y}\right)$ plane. The center corresponds to the perfect alignment of the PC. The points for which $\alpha$ or $\delta^{+}$ $+\delta^{-}$are constant form hyperbolas whose asymptotes are the $x$ and $y$ or the $X$ and $Y$ axes as represented in Fig. 5. In our setup, $X$ and $Y$ correspond to the two adjustment screws (denoted $V$ and $H$ hereafter) of the PC mount. By turning the $V$ or $H$ screws, we can move the beam up or down or right or left): the displacement of $k$ in Fig. 5 is along $X$ or $Y$. Keeping this geometry in mind, the proposed alignment procedure is as follows:

- Adjust the analyzer at $45^{\circ}$ and cancel the LI signal $\left(\mathrm{LI}_{45} \propto \delta^{+}+\delta^{-}\right)$by use of one of the two screws ( $V$ or $H)$.

- Adjust the analyzer at $0^{\circ}$ and cancel the LI signal $\left(\mathrm{LI}_{0} \propto 4 \alpha\right)$ with the other screw.

As depicted at left in Fig. 5, this leads to perfect alignment with the center of the figure in two steps. To begin by placing the analyzer at $0^{\circ}$ also allows good alignment but necessitates three steps (at right in Fig. 5). The final alignment obtained in this way is of course not absolutely perfect. The limitation comes from the precision of the cancellation of the lock-in signal which is limited by the noise level. In our experiments, after a few cycles of the above procedure, it is easy to achieve a relative precision of a few $10^{-4}$ for LI compared with the direct signal. This corresponds to $k_{x}, k_{y}$ of the order of $\sim 0.1 \mathrm{mrad}$ (see Fig. 4), which is less than the misalignment given by a fraction of one turn of the fine micrometer adjustment screw in the PC mount. Note that the alignment corresponds to $\theta^{2} \sim 10^{-8}$, a 3-order-of-magnitude increase compared with the preliminary alignment procedure. From Eqs. (8) and (11) we can estimate that this limit corresponds to a relative artifact of a few $10^{-5}$ when the pump-induced change of the probe transmission is equal to $10 \%$, which is lower than our noise level.

This procedure therefore allows us to dispense with the artifact signals that are due to misalignment of the PC. To provide an experimental demonstration of the above 

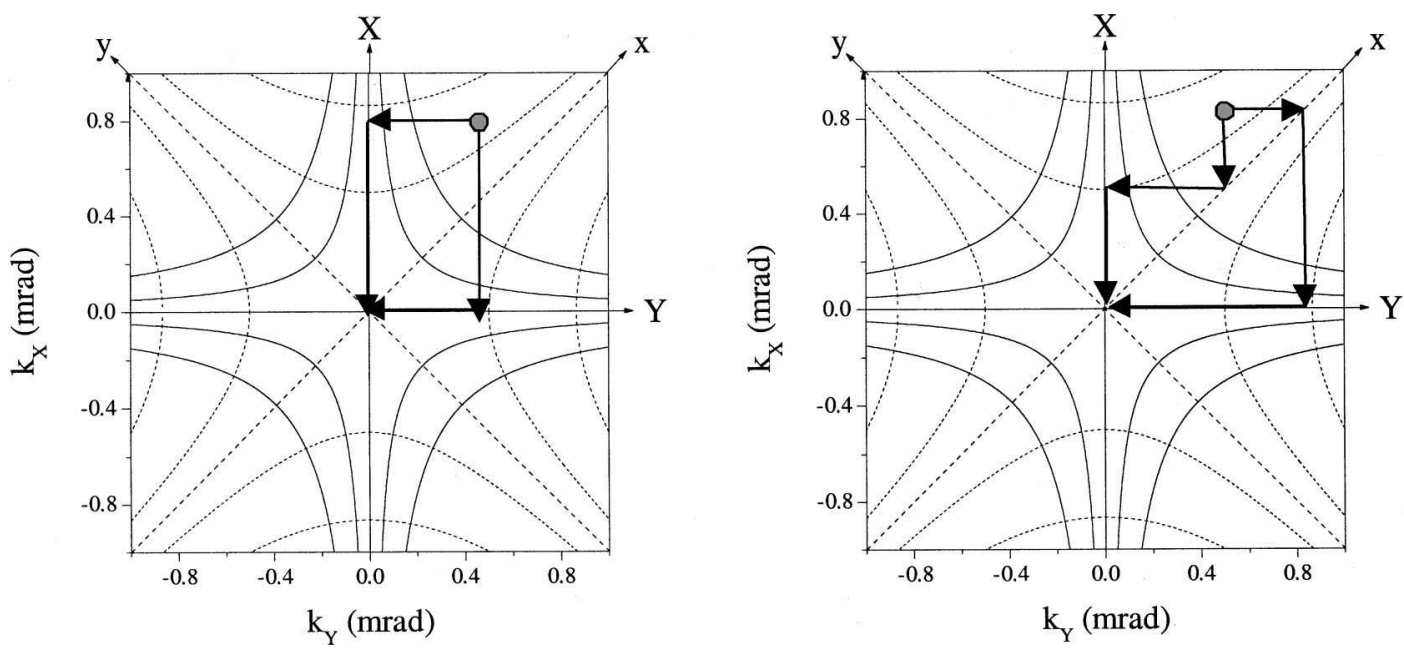

Fig. 5. Representation of the $\left(k_{X}, k_{Y}\right)$ plane. The center corresponds to a perfect alignment of the PC. Dotted curves, points at which $\mathrm{LI}_{0}(\propto 4 \alpha)$ is equal to $0, \pm 1$, and $\pm 3 \times 10^{-3}$; solid curves, same as for $\mathrm{LI}_{45}\left(\propto \delta^{+}+\delta^{-}\right)$. Figure represents the adjustment procedure when one starts with the analyzer at $45^{\circ}$ (at left) and at $0^{\circ}$ (at right).

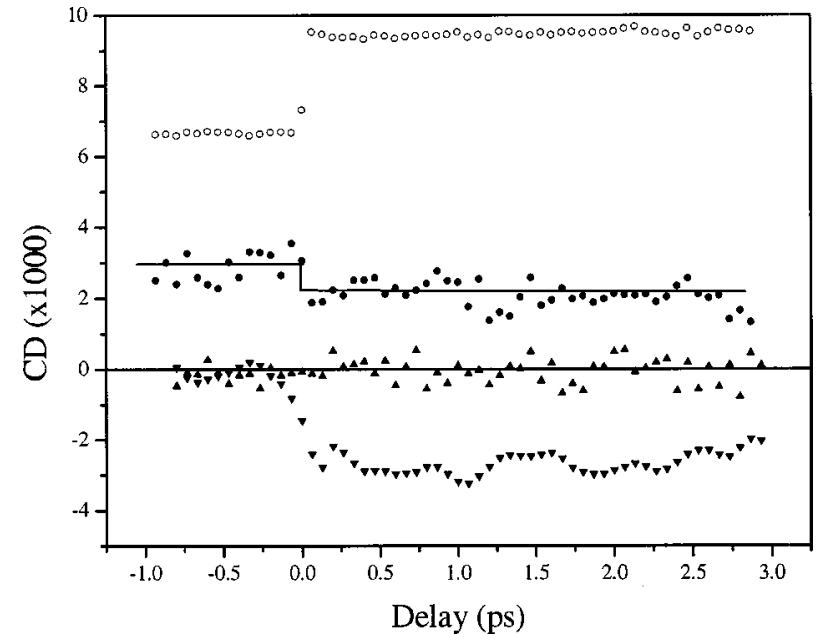

Fig. 6. Pump-probe experiment in a ruthenium-tris(bipyridyl) salt. The up and down triangles that were obtained with the racemic mixture demonstrate the consequence of a misalignment of the PC. The filled circles were obtained with the $\Delta$ enantiomer and correspond to a true pump-induced CD experiment. ${ }^{12}$ The open circles are the PMT signal (in arbitrary units), showing the onset of the absorption saturation at delay 0 .

considerations, we carried out a pump-probe experiment with a nonchiral sample [racemic mixture of a rutheniumtris(bipyridyl) salt]. We have adapted the laser characteristics to this sample ${ }^{12}$ : the pump consists of $1-\mu J$, $400-\mathrm{nm}$ pulses, and the probe wavelength is $420 \mathrm{~nm}$. The results are displayed in Fig. 6. We obtained the up triangles after following the above procedure. We performed two $0^{\circ}-45^{\circ}$ cycles for the analyzer. No residual artifact is detectable. In contrast we obtained the down triangles after we misaligned the $H$ fine adjustment screws by one turn. The corresponding misalignment of $\theta \sim 0.7 \mathrm{mrad}$ is three times smaller than the best precision obtained when we utilized the preliminary alignment with the help of the dark cross. In that case, the observed signals are just artifacts and should not be associated with a pump-induced CD signal. This again proves the necessity to go further than the usual PC alignment to obtain reliable results. Minimizing this artifact signal could appear to be sufficient to ensure an artifact-free measurement. This is only partly true because, in that case, the PC alignment would not necessarily be perfect but would provide two elliptical polarizations symmetrical compared with the pump polarization direction that would accidentally cancel the artifacts. However, in addition to the fact that such an experiment is not easier to perform than our alignment procedure, it presents two disadvantages. First, it is dependent on pump polarization. Second, a perfectly aligned PC is less prone to temperature drifts and makes the experiment more robust. We therefore believe that our procedure has the potential to be used to carry out time-resolved CD experiments with good results. As a final check, we performed the measurement in an enantiomerically pure $\Delta$-rutheniumtris(bipyridyl) salt (Fig. 6). At negative pump-probe delays, we obtained a nonzero signal that is the signature of the natural CD of this chiral molecule. The CD then drops at positive delays, a signature of the pump-induced CD, as already observed. ${ }^{12}$ This drop is clearly stronger than the noise, but one can observe that this timeresolved signal is weaker than the artifact obtained with a misalignment of one turn of a screw. This justifies the importance of the precise alignment procedure that we have devised.

\section{CONCLUSION}

We have provided an analysis of the artifacts that can appear in pump-induced CD experiments. These experiments consist of exciting a solution of chiral molecules and following the subsequent evolution of the CD. A PC is utilized as a (circular) polarization modulator, and it has been shown that a slight misalignment of the PC could induce artifacts in the pump-induced CD experiment. To control the importance of the effects and to propose solutions, we developed a calculation of the behavior of a PC when the alignment was not perfect. This calculation has allowed us to devise a simple alignment proce- 
dure that utilizes an analyzer at $0^{\circ}$ or $45^{\circ}$ of the $\mathrm{PC}$ axes. Good alignment was achieved, which improved the alignment precision by 3 orders of magnitude. The residual artifacts are well below the noise limit of our detection setup.

Thanks to our calculations, we also gained valuable information about the sensitivity of our CD measurements to several other parameters. It appears that our setup is not critically sensitive to the orientation of the PC axes with respect to the incoming probe polarization, to the applied voltages, or to the direction of the (linear) pump polarization. These features relax many of the experimental constraints, resulting in a more user-friendly setup.

This procedure permits us to utilize the time-resolved CD measurements with confidence. These measurements can be applied to many systems and are in particular well adapted to the study of the conformational dynamics of biological systems. Good candidates for the study are the hemoproteins that are both photoexcitable and display $\mathrm{CD}$ in the visible. Such experiments will be undertaken in the near future.

\section{ACKNOWLEDGMENT}

We thank M. C. Schanne-Klein and A. de Martino for their careful reading of the manuscript and the thorough discussions with regard to our conclusions.

F. Hache can be reached by e-mail at francois.hache @polytechnique.fr.

\section{REFERENCES}

1. B. Alberts, D. Bray, J. Lewis, M. Raff, K. Roberts, and J. D. Watson, Molecular Biology of the Cell (Garland Science, New York, 1994)

2. C. L. Weaver, M. Espinoza, Y. Kress, and P. Davies, "Conformational changes as one of the earliest alterations of tau in Alzheimer's disease," Neurobiol. Aging 21, 719-727 (2000).
3. G. C. Telling, P. Parchi, S. J. DeArmond, P. Cortelli, P. Montagna, R. Gabizon, J. Mastrianni, E. Lugaresi, P. Gambetti, and S. B. Prusiner, "Evidence for the conformation of the pathologic isoform of the prion protein enciphering and propagating prion diversity," Science 274, 2079-2082 (1996).

4. U. Mayor, C. R. Johnson, V. Daggett, and A. R. Fersht, "Protein folding and unfolding in microseconds to nanoseconds by experiment and simulation," Proc. Natl. Acad. Sci. U.S.A. 97, 13518-13522 (2000).

5. J. W. Lewis, R. F. Tilton, C. M. Einterz, S. J. Milder, I. D. Kuntz, and D. S. Kliger, "New technique for measuring circular dichroism changes on a nanosecond time scale: application to (carbonmonoxy) myoglobin and (carbonmonoxy) hemoglobin," J. Phys. Chem. 89, 289-294 (1985).

6. J. W. Lewis, R. A. Goldbeck, D. S. Kliger, X. Xie, R. C. Dunn, and J. D. Simon, "Time-resolved circular dichroism spectroscopy: experiment, theory and applications to biological systems," J. Phys. Chem. 96, 5243-5254 (1992).

7. S. Wenzel and V. Buss, "A split beam method for measuring time-resolved circular dichroism," Rev. Sci. Instrum. 68, 1886-1888 (1995)

8. D. W. Neyer, L. A. Rahn, D. W. Chandler, J. A. Nunes, and W. G. Tong, "Circular dichroism spectroscopy using coherent laser-induced thermal gratings," J. Am. Chem. Soc. 119, 8293-8300 (1997).

9. R. A. Goldbeck, D. B. Kim-Shapiro, and D. S. Kliger, "Fast natural and magnetic circular dichroism spectroscopy," Annu. Rev. Phys. Chem. 48, 453-479 (1997).

10. X. Xie and J. D. Simon, "Picosecond time-resolved circular dichroism spectroscopy: experimental details and applications," Rev. Sci. Instrum. 60, 2614-2627 (1989).

11. H. Mesnil, M. C. Schanne-Klein, F. Hache, M. Alexandre, G. Lemercier, and C. Andraud, "Experimental observation of nonlinear circular dichroism in a pump-probe experiment," Chem. Phys. Lett. 338, 269-276 (2001).

12. H. Mesnil, M. C. Schanne-Klein, F. Hache, M. Alexandre, G. Lemercier, and C. Andraud, "Wavelength dependence of nonlinear circular dichroism in a chiral rutheniumtris(bipyridyl) solution," Phys. Rev. A 66, 013802 (2002).

13. Y. R. Shen, The Principles of Nonlinear Optics (Wiley, New York, 1984), p. 294.

14. V. G. Dmitriev, G. G. Gurzadyan, and D. N. Nikogosyan, eds., Handbook of Nonlinear Crystals (Springer-Verlag, Berlin, 1991), p. 57.

15. M. Born and E. Wolf, Principles of Optics, 5th ed. (Pergamon, London, 1975), p. 665. 